\title{
The Intersection of Disability and Pregnancy: Risks for Maternal Morbidity and Mortality
}

\author{
Caroline Signore, MD, Maurice Davis, DHA, MPA-MHSA, \\ Candace M. Tingen, $\mathrm{PhD}$, and Alison N. Cernich, PhD
}

\begin{abstract}
It is estimated that 1 in 4 women in the United States live with a disability, and using population-based estimates, 10-12\% of women of childbearing age have a disability. There are limited data to suggest that women with disabilities experience higher rates of or risks for adverse outcomes related to pregnancy, delivery, and access to appropriate postpartum care. Research on specific disabling conditions demonstrates variable risk for syndromes that threaten the health of the mother, such as preeclampsia, infection, and coagulation disorders. Much of the literature suggests that normal, healthy pregnancy is possible but points to the need for tailored information for patients and providers about the intersection of their condition with pregnancy and specific care needs. Given the lack of systematic evidence in this area across conditions and functional impairments, more research is needed to clarify the interaction of specific disabilities with pregnancy and provide evidence-based information to the field to decrease the risks to mothers and their infants. This article will provide an overview of conditions that contribute to maternal morbidity and mortality as they relate to pregnancy in women with disabilities and provide resources to the field to further the investigation of this area.
\end{abstract}

Keywords: disability, pregnancy, maternal health, maternal mortality, morbidity

\section{Introduction}

$\mathbf{T}$ He Centers for Disease Control and Prevention note that a "disability" is any condition of the body or mind (impairment) that makes it more difficult for persons with a condition to do certain activities (activity limitation) and interact with the world around them (participation restrictions). ${ }^{1}$ According to the World Health Organization, ${ }^{2}$ disability has three dimensions: impairment in a person's body structure or function, or mental functioning (e.g., loss of limb and loss of vision); activity limitation, such as difficulty seeing, hearing, walking, or problem solving; and participation restrictions on normal daily activities, such as walking, engaging in social and recreational activities, or obtaining health care and preventive services. There are many types of disabilities, such as those that affect a person's vision, movement, thinking, remembering, learning, communicating, hearing, mental health, and social relationships. ${ }^{3}$
Nearly 61 million Americans have a disability, constituting $\sim 26 \%$ of the U.S. population. ${ }^{4}$ Among civilians living in the community, 40.6 million report a disabling condition. Of these, $28 \%$ have a hearing impairment, $19 \%$ have a vision disability, $38 \%$ have a cognitive disability, $51 \%$ have an ambulatory or motor disability, and $20 \%$ have self-care limitations. ${ }^{5}$ Approximately $12 \%$ of U.S. women of childbearing age have some type of disability. ${ }^{4}$ Although disabilities vary in their etiology and impact, they can be classified broadly based on common activity limitations. ${ }^{6}$ Physical disabilities, such as cerebral palsy and spinal cord injuries, are those associated with limits to mobility, flexibility, and dexterity; sensory disabilities include vision and hearing impairments; and intellectual and developmental disabilities (IDDs), such as Down syndrome and autism spectrum disorder, are associated with limitations in cognitive and adaptive functioning. ${ }^{7}$ In the past, both stigma associated with disability and sexuality and medical factors, including risks of medication

Eunice Kennedy Shriver National Institute of Child Health and Human Development, Bethesda, Maryland, USA.

(C) Caroline Signore et al. 2021; Published by Mary Ann Liebert, Inc. This Open Access article is distributed under the terms of the Creative Commons Attribution Noncommercial License (http://creativecommons.org/licenses/by-nc/4.0/) which permits any noncommercial use, distribution, and reproduction in any medium, provided the original author(s) and the source are cited.

Correction added on February 19, 2021 after first online publication of November 19, 2020: The article reflects Open Access, with copyright transferring to the author(s), and a Creative Commons Attribution Noncommercial License (CC-BY-NC) added (http:// creativecommons.org/licenses/by-nc/4.0/). 
use in pregnancy, limited childbearing in women with disabilities (WWDs). ${ }^{8}$ However, with medical advances and increased recognition of the reproductive rights of persons with disabilities, more WWDs now experience pregnancy. ${ }^{7}$

\section{Pregnancy and Reproductive Health in WWDs}

According to analyses of Medical Expenditure Panel Survey data, rates of pregnancy may differ across disability type: women with multiple limitations have the lowest proportion of pregnancies, and women with physical disabilities have the highest proportion. ${ }^{9}$ Despite the size of the disability population, the sexual and reproductive health needs of this population largely have been ignored. ${ }^{9}$ Only certain types of disabilities interfere with fecundity: the ability to conceive, in women, particularly women with cognitive disabilities. ${ }^{10}$ Reproductive health, sexual health, and sexuality in people with disabilities historically have been stigmatized, but they are important components of wellness for all women. WWDs are as likely as their nondisabled peers to desire pregnancy (61\% and $60 \%$, respectively), but fewer intend to have a baby in the future $\left(43 \%\right.$ and $50 \%$, respectively). ${ }^{11}$ Pregnancy rates among WWDs have increased in recent years and are similar to the pregnancy rates of women without disabilities in the same age and income groups. ${ }^{12,13}$

Despite these statistics, WWDs remain at a heightened risk for pregnancy-related health complications. ${ }^{9}$ They face challenges accessing health care and support before, during, and after pregnancy, which adds to these health disparities. ${ }^{14}$ WWDs may encounter negative experiences with providers who doubt their ability to become pregnant, carry the baby to term, deliver safely, and/or care for the newborn. ${ }^{12}$ WWDs also encounter negative attitudes toward pregnancy and parenting from many sources, not only from health care providers but also from the public. ${ }^{14}$ This negativity can have wide-ranging effects, including increased stress (with the associated pregnancy health risk) and a hesitancy to seek care. In fact, many WWDs do not seek preconception care, and some even forgo prenatal care because of negative reactions from providers. ${ }^{8}$ In a systematic review and meta-analysis to examine the association between maternal disabilities and risk for perinatal complications, it was noted that women with sensory, intellectual, and developmental disabilities had an elevated risk for gestational diabetes and hypertensive disorders (gestational hypertension, eclampsia, and/or preeclampsia) and a significant risk for cesarean delivery. ${ }^{7}$ Overall, these findings suggest the need to better support WWDs during the prenatal period and to produce high-quality research to further explore factors that may contribute to their increased risk for perinatal complications. What follows is a review of the specific pregnancy outcomes and complications experienced by WWDs as documented by a thorough literature review conducted through PubMed and a review of federal resources available online.

\section{Maternal Morbidity and Mortality Among WWDs}

The overall number of pregnancy-related deaths in the United States is 16.9 deaths per 100,000 live births in 2016, which is equivalent to $\sim 700$ women per year. ${ }^{7}$ The top specific causes of maternal death for all groups in the United States are infection $(13.3 \%)$, hemorrhage $(11.1 \%)$, cardio- myopathy $(11.1 \%)$, thrombotic pulmonary or other embolisms $(9.2 \%)$, hypertensive disorders of pregnancy $(7.8 \%)$, cerebrovascular accidents (7.2\%), amniotic fluid embolism $(5.4 \%)$, and anesthesia complications $(0.4 \%)$, with the catchall of "other conditions" (other cardiovascular conditions [15.3\%], other noncardiovascular conditions [13.3\%], and unknown causes [5.8\%]) accounting for the remaining deaths. ${ }^{7}$ All cardiovascular conditions together (cardiomyopathy, cerebrovascular accidents, and other cardiovascular conditions), therefore, account for $\sim 33 \%$ of all pregnancyrelated deaths. However, these aggregated numbers obscure considerable disparities in the causes of death, which vary not only by the timing related to pregnancy, but also by the race, ethnicity, and disability status of the women.

It is well documented that WWDs experience persistent disparities in health care access and outcomes compared with nondisabled women. ${ }^{15}$ Social determinants of health and risk factors for poor pregnancy outcomes are more prevalent in the disability community as a whole, with lower educational attainment; higher poverty rates; higher rates of social isolation $^{4,16}$; and higher rates of medical risk factors, such as obesity, diabetes, stress, depression, smoking, and alcohol and/or substance use. ${ }^{4,17}$ WWDs who become pregnant have poorer health and higher sociodemographic risk factors, in general, than women without disabilities. ${ }^{18-20}$ In a U.S. population-based study of delivery hospitalizations among women with $(n=1,897)$ and without $(n=4,194,938)$ IDDs between 2007 and 2011, women with IDDs were more likely to be black, young, publicly insured, low income, and from rural areas, and were nearly three times as likely to have one or more comorbidities (72\% vs. $23 \%){ }^{15}$ Using data from California, Darney et al. ${ }^{21}$ found that pregnant WWDs were more likely to have hypertension, and women with vision disabilities had higher rates of pregestational diabetes than nondisabled pregnant women. Mitra et al. ${ }^{22}$ reported that women with hearing loss were significantly more likely than women without hearing loss to report comorbidity during pregnancy.

Given the risk factors WWDs bring into pregnancy, there likely is an increased theoretical risk of maternal mortality in this population. In addition, all-cause mortality among community-dwelling adults with any disability is increased compared with adults without a disability (adjusted hazard ratio $=1.51,95 \%$ confidence interval $[\mathrm{CI}][1.45-1.57]$ ), with a greater magnitude of the association between disability and death in young and middle-aged adults (18-64 years). ${ }^{23}$ The leading cause of death in the population was heart disease, and mortality was highest for movement disability, followed by mental/substance use impairment and sensory disability. However, definitive data related to maternal mortality are lacking. Mueller et al. ${ }^{24}$ used Washington state-linked hospital discharge and vital statistics data $(n=745,000$ women of reproductive age) to examine pregnancy outcomes among women with IDDs, but there were too few deaths for a meaningful analysis. One study examined maternal deaths within 2 years of pregnancy in women with paralysis due to spinal cord injury, spina bifida, or other conditions, and found an overall risk of 19.23 (95\% CI [5.53-66.88]); however, most of the deaths were neither pregnancy related nor disability related. ${ }^{25}$

In recent years, however, there has been a growing interest in and number of publications on maternal outcomes in WWDs, furthering our knowledge regarding the impact of a disabling condition(s) on pregnancy morbidity and the outcomes that are 
associated with high mortality. In general, WWDs enter pregnancy in poorer health than nondisabled people. Using National Health Interview Survey data, Iezzoni et al. ${ }^{20}$ found that $29 \%$ of pregnant women with chronic physical disabilities reported fair or poor health, compared with just $3 \%$ of nondisabled women. The most prevalent causes of maternal morbidity and mortality are considered hereunder within a framework of how a maternal disability may affect risk.

\section{Infection}

Lower urinary tract infections (UTIs) are the most common infections during pregnancy, and physiological changes to the urinary system during pregnancy increase the risk of ascending infection (pyelonephritis). ${ }^{26}$ Asymptomatic bacteriuria is present in $2 \%-7 \%$ of the general population of pregnant women but approaches $100 \%$ for women with mobility disorders and neurogenic bladders who depend on a permanent indwelling urinary catheter. Untreated bacteriuria may lead to symptomatic UTI in $25 \%$ of pregnant women. In one small study of 22 pregnant women with a spinal cord injury, the incidence of symptomatic UTI was $100 \% .{ }^{27}$ Increased incidence of UTIs also has been reported among women with multiple sclerosis (MS) (adjusted rate ratio $[\mathrm{aRR}]=1.8[1.2-2.6]){ }^{28}$

Pyelonephritis affects $0.5 \%$ of pregnant women ${ }^{29}$ and is the leading cause of sepsis syndrome during pregnancy. In a retrospective cohort study using data from Washington state, the risk of contracting a combined outcome UTI or pyelonephritis increased markedly among pregnant women with paralysis from spinal cord disorders, compared with women without paralysis $(\mathrm{RR}=26.43$ [13.97-49.99] $){ }^{25}$ Morton et al. ${ }^{30}$ reported similar results in a small retrospective cohort study of 34 pregnancies in women with moderate-to-severe physical disabilities compared with nondisabled controls. In that study, pyelonephritis occurred in 5 of 34 (15\%) WWDs, and urosepsis occurred in 1 of 34 (3\%) WWDs.

Cesarean delivery increases the risk of postpartum infections, including endometritis and infectious wound complications. Because several reports suggest that cesarean delivery is more common among WWDs, ${ }^{21}$ including both physical ${ }^{25,28}$ and IDDs, ${ }^{17,24,31}$ this may put them at increased risk for infectious morbidity. Morton et al. ${ }^{30}$ observed more cases of endometritis among WWDs than among nondisabled women (12\% vs. $3 \%$ ), but this difference was not statistically significant.

Some disabilities are associated with respiratory impairment, ${ }^{32}$ which could increase the incidence and/or severity of perinatally acquired pneumonia. A study of comorbid conditions among pregnant women with chronic physical disabilities found that, among women who reported arthritis as their disabling condition, for example, many also reported asthma $(31 \%)$ or chronic obstructive pulmonary disease $(21 \%){ }^{33}$ A study of pregnant women with and without MS showed a higher incidence of lower respiratory tract infection ( $5.2 \%$ vs. $3.9 \%$, respectively), but this difference did not reach statistical significance. ${ }^{34}$

Houtchens et al., ${ }^{32}$ using an administrative claims database, reported that women with MS had a higher risk of infection complicating pregnancy than women without MS. Using other databases, MacDonald et al. ${ }^{34}$ also found a higher risk of infection among women with MS, including a higher risk of genitourinary infections $(\mathrm{aRR}=1.26$ [1.17-1.36]) and upper respiratory tract infections $(\mathrm{aRR}=1.33[1.20-1.45])$.

\section{Hemorrhage}

Certain risk factors for obstetric hemorrhage are more common among pregnant WWDs. As already noted, cesarean delivery is more common in WWDs and is a known risk factor for postpartum hemorrhage. Similarly, obesity and preeclampsia/eclampsia are risk factors for hemorrhage and are increased in WWDs. Brown et al. ${ }^{19}$ conducted a retrospective cohort study of 3,932 women with IDDs and 382,774 controls and found a significantly increased risk of preeclampsia $(\mathrm{aRR}=1.47)$, peripartum hemorrhage $(\mathrm{aRR}=$ 1.30), abruptio placentae, and cesarean delivery. Studies of pregnant women with MS, however, showed no increased risk for hemorrhagical complications. ${ }^{28,32,34}$

\section{Cardiomyopathy}

Pregnant WWDs tend to have certain risk factors for the development of cardiomyopathy. These include preconception diabetes and chronic hypertension (CHTN), smoking, and obesity. ${ }^{33}$ The incidence of peripartum cardiomyopathy in WWDs is not known.

\section{Thromboembolic Disease}

The risk of developing deep venous thrombosis and pulmonary embolism increases during pregnancy. This risk is exacerbated in women with mobility disorders, especially those with lower limb paralysis who are nonambulatory and use wheelchairs. Crane et al. ${ }^{25}$ reported a greater than ninefold increased risk of venous thromboembolism/pulmonary embolism during pregnancy among women with paralytic spinal cord disorders compared with controls $(\mathrm{RR}=9.16$ [2.17-38.60]). Women with IDDs also may be at higher risk. In one Canadian study, ${ }^{19}$ venous thromboembolism occurred more often in women with IDDs than in control pregnancies $(\mathrm{aRR}=1.60$ [1.17-2.19]). Other risk factors for thromboembolic disease include obesity, smoking, and cesarean delivery, all of which are more prevalent among WWDs.

\section{CHTN and Hypertensive Disorders of Pregnancy}

In recent years, $\mathrm{CHTN}$ has become one of the most common serious complications of pregnancy. ${ }^{26} \mathrm{CHTN}$ increases the risk for adverse pregnancy outcomes, including preeclampsia, cardiomyopathy and heart failure, and peripartum stroke. Pregnant WWDs are more likely than nondisabled women to have CHTN, among other comorbidities. ${ }^{15,21,22}$ Brown et al., ${ }^{31}$ in a population-based Canadian cohort study, found that, compared with pregnant women without IDDs, women with IDDs had a significantly increased risk of preeclampsia $(\mathrm{aRR}=1.47[1.11-1.93])$.

\section{Cerebrovascular Accidents}

Pregnant women may have impairments as a result of a prior stroke. As with other conditions associated with maternal morbidity and mortality, some risk factors for peripartum stroke are more common among pregnant WWDs: obesity, heart disease, CHTN, diabetes, and smoking. ${ }^{19,32,35}$ The most common risk factors for cerebrovascular accidents are pregnancy-associated hypertensive disorders. ${ }^{26}$ As already noted, an increased risk of preeclampsia/eclampsia has been shown among women with IDDs. ${ }^{19}$ 


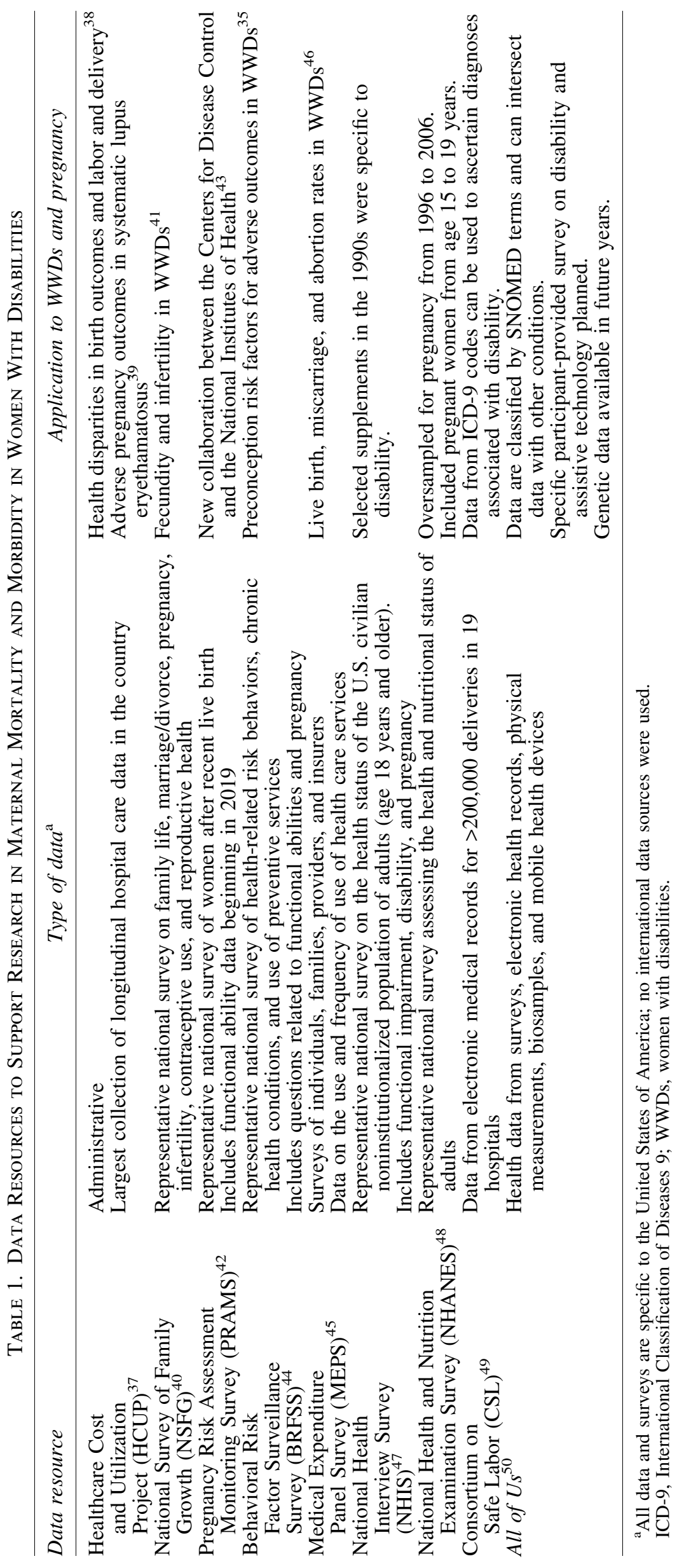




\section{Amniotic Fluid Embolism}

This potentially catastrophic complication of delivery is difficult to predict and is rare, with an estimated incidence of 2.2-7.7 cases per 100,000 deliveries. ${ }^{36}$ Few studies of WWDs examine rates of this outcome. One study of 2,115 women with MS and 2,115 women without MS detected no increased risk, but this study was underpowered for such a rare outcome. ${ }^{32}$

\section{Other Cardiovascular Diseases}

Data on other cardiovascular outcomes among pregnant WWDs are scant. Brown et al. found an increased risk of "systemic maternal complications" in a population-based study of women with IDDs in Ontario, Canada. ${ }^{19}$ This composite outcome, consisting of cardiomyopathy, cardiac arrest, cardiac failure, cerebrovascular disease, myocardial infarction, disseminated intravascular coagulation, pulmonary edema, acute respiratory distress syndrome, complications of anesthesia, acute renal failure, hepatic failure, and status epilepticus, was $>2.5$ times more likely in women with IDDs than in women without IDDs $(\mathrm{aRR}=2.59$ [1.64-4.11]). This finding appeared to be driven by a statistically significant threefold increased risk of pulmonary edema among women with IDDs.

\section{Comment}

Resources for research in maternal mortality and morbidity in WWDs

Given the need to further investigate the intersection of maternal mortality and morbidity with specific types of disability, specific resources can be used to generate or explore hypotheses. These include available administrative data resources, data and biospecimens from research cohorts that are available on data-sharing platforms, and information from crowd-sourcing efforts sponsored by research organizations. Table 1 summarizes some of these resources and the availability of data, with links to sources and requirements for data use.

\section{Conclusions}

The historic neglect of sexual and reproductive health in WWDs has led to a long-standing dearth of information on the prepregnancy, pregnancy, labor and delivery, and postpartum complications that these women may experience. However, investigators are beginning to understand the relevance of these outcomes for a population of women who historically were underrepresented in the gynecological and obstetric literature. As more prospective studies, national surveys, administrative data sources, and large-scale data initiatives recognize the need to include WWDs, data are becoming available to better understand outcomes of interest in these populations. Moreover, understanding the specific risks that accompany certain diagnostic conditions or types of disability will contribute to the evidence informing clinical practice guidelines and preventive health interventions in WWDs. Finally, though challenging, specific studies related to maternal mortality in this population are needed to better inform prenatal and postpartum care.

The literature reviewed here makes clear that there are specific risks of pregnancy for WWDs, especially as they relate to infection or cardiovascular conditions, but additional considerations are warranted in individuals with specific conditions and comorbidities. Some portion of these increased risks can be attributed to poor prepregnancy health and the impact of social determinants of health for WWDs. Study to further improve the management of general health and wellness in this population will contribute to better pregnancy and birth outcomes. Most importantly, though, WWDs can have healthy pregnancies with normal birth outcomes, and many want to have children. Providers can and should foster healthy pregnancies in those women who would like to have children and should partner with them and other health providers to enable them to participate fully in their desired roles.

\section{Acknowledgments}

The authors acknowledge the efforts and coordination of colleagues at the Office of Research on Women's Health for this special issue, and our colleagues in the federal and extramural community who conducted the research referenced.

\section{Author Disclosure Statement}

No competing financial interests exist.

\section{Funding Information}

No funding was received for this article.

\section{References}

1. Centers for Disease Control (CDC). Disability and health overview: Impairments, activity limitations, and participation restrictions. Available at: https://www.cdc.gov/ ncbddd/disabilityandhealth/disability.html Accessed May $10,2020$.

2. World Health Organization (WHO). WHO Global Disability Action Plan 2014-2021: Better health for all people with a disability, 2015. Available at: https://apps.who.int/ iris/handle/10665/199544 Accessed May 10, 2020.

3. WHO. International classification of functioning, disability and health (ICF). Geneva, Switzerland: WHO Press, 2001.

4. Courtney-Long EA, Carrol DD, Zhang QC, et al. Prevalence of disability and disability type among adults. MMWR Morb Mortal Wkly Rep 2015;64:777-783.

5. Institute on Disability/UCED. 2019 Annual Disability Statistics Compendium. Durham, NH: University of New Hampshire Press, 2020.

6. Wen X, Fortune N. The definition and prevalence of physical disability in Australia. Canberra, Australia: Australian Institute of Health and Welfare, 1999.

7. Tarasoff L, Ravindran S, Malik H, Salaeva D, Brown H. Maternal disabilty and risk for pregnancy, delivery, and postpartum complications: A systematic review and metaanalysis. Am J Obstet Gynecol 2020;222:27.e1-27.e32.

8. Smeltzer SC. Reproductive decision making in women with multiple sclerosis. J Neurosci Nurs 2002;34:145-157.

9. Horner-Johnson W, Darney BG, Kulkarni-Rajasekhara S, Quigley B, Caughey AB. Pregnancy among U.S. women: Differences by presence, type, and complexity of disability. Am J Obstet Gynecol 2016;214:529.e1-529.e9.

10. Zhang Y, McLain A, Davis B, McDermott S. Fecundity and infertility among women with disabilities in the United States. J Womens Health (Larchmt) 2019;28:934-940. 
11. Signore C, Spong CY, Krotoski D, Shinowara NL, Blackwell SC. Pregnancy in women with physical disabilities. Obstet Gynecol 2011;117:935-947.

12. Bloom T, Mosher W, Alhusen J, Lantos H, Hughes R. Fertility desires and intentions among U.S. women by disability status: Findings from the 2011-2013 National Survey of Family Growth. Matern Child Health 2017;21: 1606-1615.

13. National Council on Disability. Rocking the cradle: Ensuring the rights of parents with disabilities and their children. Washington, DC: National Council on Disability, 2012. Available at: https://ncd.gov/publications/2012/ Sep272012/ Accessed May 10, 2020.

14. Signore C. Eliminating inequities for women with disabilities: Reproductive and sexual health. Washington, DC: American Psychological Association, 2016.

15. Akobirshoev I, Parish SL, Mitra M, Rosenthal E. Birth outcomes among US women with intellectual and developmental disabilities. Disabil Health J 2017;10:406412.

16. Petersen EE, Davis NL, Goodman D, et al. Racial/ethnic disparities in pregnancy-related deaths-United States, 2007-2016. MMWR Morb Mortal Wkly Rep 2019;68:762765.

17. Mitra M, Akobirshoev I, Parish SL, Valentine A, Clements KM, Moore Simas TA. Postpartum emergency department use among women with intellectual and developmental disabilities: A retrospective cohort study. J Epidemiol Community Health 2019;73:557-563.

18. MacDonald SC, McElrath TF, Hernandez-Diaz S. Pregnancy outcomes in women with multiple sclerosis. Am J Epidemiol 2019;188:57-66.

19. Brown HK, Cobigo V, Lunsky Y, Vigod SN. Maternal and offspring outcomes in women with intellectual and developmental disabilities: A population-based cohort study. BJOG 2017;124:757-765.

20. Iezzoni LI, Yu J, Wint AJ, Smeltzer SC, Ecker JL. General health, health conditions, and current pregnancy among U.S. women with and without chronic physical disabilities. Disabil Health J 2014;7:181-188.

21. Darney BG, Biel FM, Quigley BP, Caughey AB, HornerJohnson W. Primary cesarean delivery patterns among women with physical, sensory, or intellectual disabilities. Womens Health Issues 2017;27:336-344.

22. Mitra M, Akobirshoev I, McKee MM, Iezzoni LI. Birth outcomes among U.S. women with hearing loss. Am J Prev Med 2016;51:865-873.

23. Forman-Hoffman VL, Ault KL, Anderson WL, et al. Disability status, mortality, and leading causes of death in the United States community population. Med Care 2015;53: 346-354.

24. Mueller BA, Crane D, Doody DR, Stuart SN, Schiff MA. Pregnancy course, infant outcomes, rehospitalization, and mortality among women with intellectual disability. Disabil Health J 2019;12:452-459.

25. Crane DA, Doody DR, Schiff MA, Mueller BA. Pregnancy outcomes in women with spinal cord injuries: A population-based study. PM R 2019;11:795-806.

26. Cunningham F, Leveno KL, Bloom SL, et al. Williams obstetrics, 24th ed. New York, NY: McGraw-Hill Education/Medical, 2014.

27. Cross LL, Meythaler JM, Tuel SM, Cross AL. Pregnancy, labor and delivery post spinal cord injury. Paraplegia 1992; 30:890-902.
28. Fong A, Chau CT, Quant C, Duffy J, Pan D, Ogunyemi DA. Multiple sclerosis in pregnancy: Prevalence, sociodemographic features, and obstetrical outcomes. J Matern Fetal Neonatal Med 2018;31:382-387.

29. Wing DA, Fassett MJ, Getahun D. Acute pyelonephritis in pregnancy: An 18-year retrospective analysis. Am J Obstet Gynecol 2014;210:219.e1-219.e6.

30. Morton C, Le JT, Shahbandar L, Hammond C, Murphy EA, Kirschner KL. Pregnancy outcomes of women with physical disabilities: A matched cohort study. PM R 2013;5: 90-98.

31. Brown HK, Cobigo V, Lunsky Y, Dennis CL, Vigod S. Perinatal health of women with intellectual and developmental disabilities and comorbid mental illness. Can J Psychiatry 2016;61:714-723.

32. Houtchens MK, Edwards NC, Schneider G, Stern K, Phillips AL. Pregnancy rates and outcomes in women with and without MS in the United States. Neurology 2018;91: e1559-e1569.

33. Iezzoni LI, Yu J, Wint AJ, Smeltzer SC, Ecker JL. Conditions causing disability and current pregnancy among US women with chronic physical disabilities. Med Care 2014; 52:20-25.

34. MacDonald SC, McElrath TF, Hernandez-Diaz S. Use and safety of disease-modifying therapy in pregnant women with multiple sclerosis. Pharmacoepidemiol Drug Saf 2019; 28:556-560.

35. Mitra M, Clements KM, Zhang J, Smith LD. Disparities in adverse preconception risk factors between women with and without disabilities. Matern Child Health J 2016;20: 507-515.

36. Skolnik S, Ioscovich A, Eidelman LA, et al. Anesthetic management of amniotic fluid embolism-A multi-center, retrospective, cohort study. J Matern Fetal Neonatal Med 2019;32:1262-1266.

37. HCUP Home. Healthcare Cost and Utilization Project (HCUP). Rockville, MD: Agency for Healthcare Research and Quality, 2020. Available at: www.hcup-us.ahrq.gov/ home.jsp Accessed May 10, 2020.

38. Akobirshoev I, Mitra M, Parish SL, Moore Simas TA, Dembo R, Ncube CN. Racial and ethnic disparities in birth outcomes and labour and delivery-related charges among women with intellectual and developmental disabilities. J Intellect Disabil Res 2019;63:313-326.

39. Ling N, Lawson E, von Scheven E. Adverse pregnancy outcomes in adolescents and young women with systemic lupus erythematosus: A national estimate. Pediatr Rheumatol Online J 2018;16:26.

40. National Center for Health Statistics. National Survey of Family Growth, 2020. Available at: https://www.cdc.gov/ nchs/nsfg/index.htm Accessed May 10, 2020.

41. Division of Reproductive Health, National Center for Chronic Disease Prevention and Health Promotion, Centers for Disease Control. PRAMS: Pregnancy Risk Assessment Monitoring System, 2020. Available at: https://www.cdc .gov/prams/index.htm Accessed May 10, 2020.

42. D'Angelo DV, Cernich A, Harrison L, et al. Disability and pregnancy: A cross-federal agency collaboration to collect population-based data about experiences around the time of pregnancy. J Womens Health (Larchmt) 2020;29:291296.

43. National Center for Chronic Disease Prevention and Health Promotion, Division of Population Health. Behavioral Risk Factor Surveillance System. 2020. Available 
at: https://www.cdc.gov/brfss/index.html Accessed May $10,2020$.

44. Agency for Healthcare Research and Quality. Medical Expenditure Panel Survey, 2020. Available at: https://www .meps.ahrq.gov/mepsweb/ Accessed May 10, 2020.

45. Horner-Johnson W, Kulkarni-Rajasekhara S, Darney BG, Dissanayake M, Caughey AB. Live birth, miscarriage, and abortion among U.S. women with and without disabilities. Disabil Health J 2017;10:382-386.

46. National Center for Health Statistics. National Health Interview Survey, 2020. Available at: https://www.cdc.gov/ nchs/nhis/index.htm Accessed May 10, 2020.

47. National Center for Health Statistics. National Health and Nutrition Examination Survey, 2020. Available at: https:// www.cdc.gov/nchs/nhanes/index.htm Accessed May 10, 2020.

48. Zhang J, Troendle J, Reddy UM, et al. Contemporary cesarean delivery practice in the United States. Am J Obstet Gynecol 2010;203:326.e1-326.e10.
49. National Institutes of Health. All of Us Research Program, 2020. Available at: https://allofus.nih.gov/ Accessed May $25,2020$.

50. National Institutes of Health All of Us Research Program. Opportunities for Researchers. Available at: https://allofus .nih.gov/get-involved/opportunities-researchers Accessed November 13, 2020.

Address correspondence to: Caroline Signore, MD Eunice Kennedy Shriver National Institute of Child Health and Human Development $6710 B$ Rockledge Drive MSC 7002 । Room 2320

Bethesda, MD 20817

USA

E-mail: signorec@mail.nih.gov 